\title{
Probing Dark Energy in the Accelerating Universe with SNAP
}

\author{
Michael Schubnell (for the SNAP Collaboration) ${ }^{1}$
}

\author{
Physics Department, University of Michigan, Ann Arbor, MI 48109
}

\begin{abstract}
It has now been firmly established that the Universe is expanding at an accelerated rate, driven by a presently unknown form of dark energy that appears to dominate our Universe today. A dedicated satellite mission has been designed to precisely map out the cosmological expansion history of the Universe and thereby determine the properties of the dark energy. The SuperNova / Acceleration Probe (SNAP) will study thousands of distant supernovae, each with unprecedented precision, using a 2-meter aperture telescope with a wide field, large-area optical-to-near-IR imager and high-throughput spectrograph. SNAP can not only determine the amount of dark energy with high precision, but test the nature of the dark energy by examining how its equation of state evolves. The images produced by SNAP will have an unprecedented combination of depth, solid-angle, angular resolution, and temporal sampling and will provide a rich program of auxiliary science.
\end{abstract}

\section{INTRODUCTION}

Recent measurements of luminosity distance versus redshift of nearby Type Ia supernovae by the Supernova Cosmology Project and the High-z Supernova Team have determined that the expansion of the Universe is accelerating $[1,2]$. Furthermore, the results constrain the mass density, $\Omega_{M}$, and the density of an unknown form of negative pressure energy, $\Omega_{\Lambda}$, characterized by an equation of state $w \equiv p / \rho<-1 / 3$ causing the acceleration. This additional energy component, coined dark energy, appears to dominate energy density and dynamics of the Universe at the present epoch.

The evidence for dark energy is in remarkable concordance with other observations. Measurements of small scale fluctuations in the cosmic microwave background (CMB) radiation support the supernova results and have determined that the Universe is nearly flat [3]. Observations of galaxy clustering [4] have shown that the fraction of the critical density consisting of matter is $\Omega_{M} \approx 0.3$, also consistent with the results obtained from the supernova measurements (Figure 1). Combined, these results strongly suggest that at the present epoch - at least $70 \%$ of the Universe's density is in the form of dark energy and only approximately $30 \%$ in some form of matter (which is mostly dark).

In its simplest form, dark energy might well be Einstein's cosmological constant in the form of a vacuum energy but numerous other theories have been proposed including the possibility of slowly evolving scalar fields (so-called quintessence models $[5,6]$.) When combined with CMB and galaxy cluster measurements, a tight bound on the dark energy equation of state $w$ can be extracted (assuming it is constant over the expansion

\footnotetext{
${ }^{1}$ for a list of SNAP collaboration members see http://snap.lbl.gov
} 

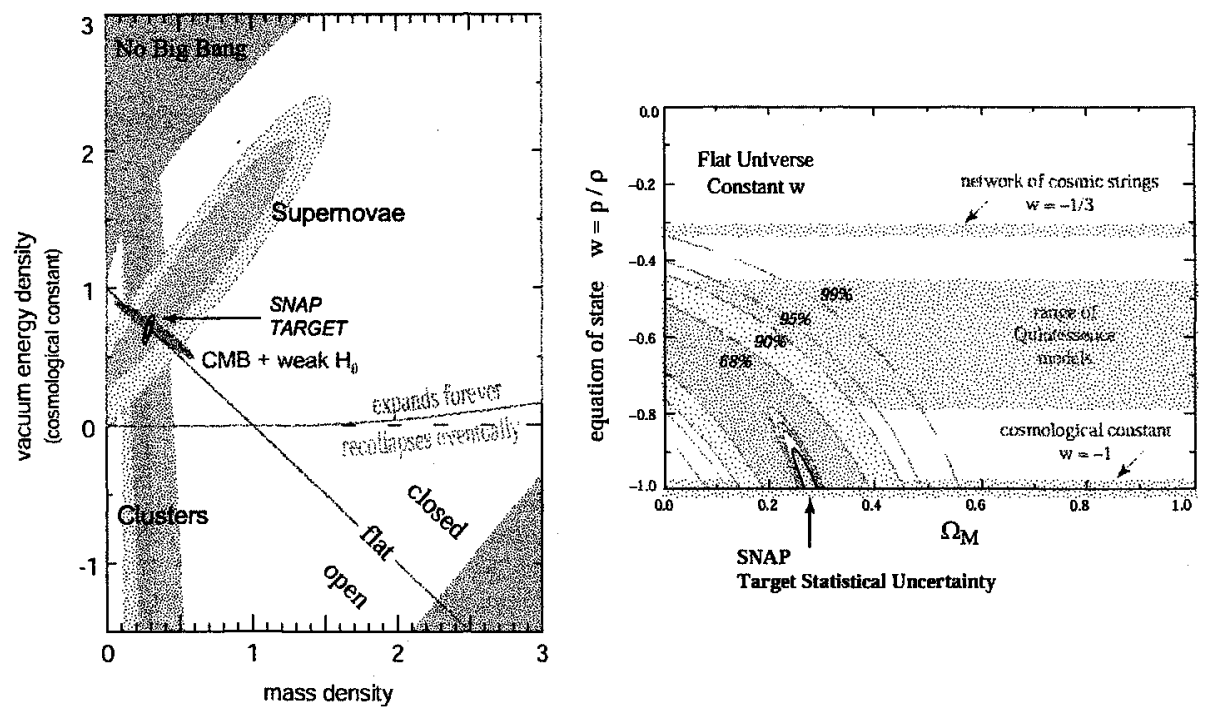

FIGURE 1. Left figure - Confidence regions in the $\Omega_{\Lambda}-\Omega_{M}$ plane for supernovae [1], galaxy cluster [4], and CMB [3] data. The consistent overlap is compelling evidence for a geometrically flat, dark energy dominated Universe. Also shown (in both figures) is the expected confidence region from the SNAP satellite for a flat $\Omega_{M}=0.28$ Universe. Right figure - Constraints on the equation-of-state parameter $w$ from the Supernova Cosmology Project [1]. Shown are confidence regions in the $\Omega_{\Lambda}-w$ plane for an energy density component $\Omega_{\Lambda}$ characterized by $w=p / \rho$. If the dark energy is Einstein's cosmological constant, then $w=-1$. Also shown are $w$ predictions for other dark energy models.

time, i.e. $d w / d z=0$ ). Current constaints on $w$ are consistent with dark energy being a cosmological constant but also allow for a wide range of alternate models, including those with a time dependent value of $w$ (Figure 1).

A precise measurement of dark energy properties requires a much larger data set of supernovae than currently available and a significant improvement of the systematic uncertainties in the measurements over current experiments. A definitive program to study dark energy with supernovae must provide a high degree of statistical and systematic rigor [7]. Furthermore, the greatest sensitivity to cosmological parameters is obtained with measurements extending from the present epoch of acceleration into the matter dominated deceleration phase [8]. Because measurements of the highly redshifted light from very distant supernovae require sensitivity into the near infrared (NIR), such a program can only be achieved in space, unhindered by absorption in the earth's atmosphere.

\section{SATELLITE AND MISSION}

The primary goal of the SNAP mission is to measure cosmological parameters with a precision that will allow to distinguish between different dark energy models. For this supernova observations provide a proven and well understood cosmological tool. The 

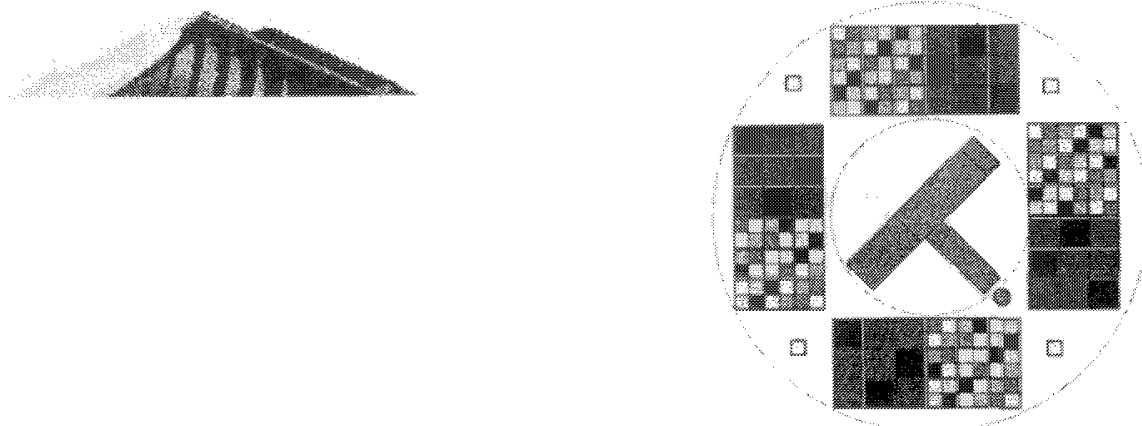

FIGURE 2. Left figure - Cross-sectional view of the SNAP satellite. Right figure - The SNAP focal plane. For detailed description of satellite and anstrument see $[9,10]$

essential measurement for this purpose is a comparison of luminosity distance to redshift providing information on the scale size as a function of expansion time. With a precisely calibrated data set of several thousand Type Ia SNe with $z=0.1-1.7$ the expansion history of the Universe can be reconstructed back to more than $70 \%$ of its age.

It has been shown that Type Ia supernovae have uniform peak $B$-band brightness when their light curves are corrected for a stretch factor which describes the relation between absolute brightness and explosion [1]. However, to fully standardize the SN peak brightness, a variety of additional observations must be made. Color measurements throughout the light curve for instance provide constraints on host-galaxy environment and galactic extinction and spectra obtained near maximum brightness allow identification of the explosion as a Type Ia through characteristic features (e.g. Sill at $6150 \AA$ ).

The SNAP satellite and mission design has been optimized for efficient supernova detection and high quality follow-up measurements. The combination of a three mirror 2 -meter telescope and $\mathrm{a} \approx 600$ million pixel optical to near infrared imager with a large 0.7 square degree field of view will allow discovery and follow up of many supernovae at once. The imaging system comprises 36 large format $(3.5 \mathrm{k} \times 3.5 \mathrm{k}) \mathrm{CCDs}$ and the same number of $2 \mathrm{k} \times 2 \mathrm{k} \mathrm{HgCdTe}$ infrared sensors. Both the CCDs and the NIR detectors are placed in four symmetric $3 \times 3$ arrangements as shown in Figure 2. Both the imager and a low resolution $(\mathrm{R} \approx 100)$ high-throughput spectrograph cover the waveband from 350 to $1700 \mathrm{~nm}$, allowing detailed characterization of supernovae out to $z=1.7$. This deep reach in redshift is essential to the mission as it will allow to resolve degeneracies in cosmological parameters and to discriminate between models of dark energy.

Nine special filters fixed above the imaging sensors will provide overlapping redshifted $B$-band coverage from $350-1700 \mathrm{~nm}$. As SNAP repeatedly steps across its target fields in the north and south ecliptic poles, every supernova will be seen in every filter in both the visible and NIR. Because of their larger linear size, each NIR filter will be visited with twice the exposure time of the visible filters. This, combined with the time-dilated light curve, will ensure that Type Ia supernovae out to redshift 1.7 will be detected with a $\mathrm{S} / \mathrm{N}>6$ at least 2 magnitudes below peak brightness $[9,10]$. 


\section{SNAP SCIENCE}

SNAP will conduct two primary surveys, a $\sim 15$ square degree ultra-deep $\left(m_{A B} \sim 30\right.$ for point sources) supernova survey, and a $\sim 300$ square degree deep $\left(m_{A B} \sim 27.8\right.$ for point sources) weak lensing survey. With this wealth of detailed data, SNAP will construct a Hubble diagram with unprecedented control over systematic uncertainties, addressing all known and proposed sources of error. The first goal is to provide precision measurements of the cosmological parameters: the matter density, $\Omega_{M}$, will be measured to \pm 0.02 , while $\Omega_{\Lambda}$, and the curvature parameter, $\Omega_{k}$, will both be determined to an accuracy of \pm 0.04 . The SNAP measurements will be largely orthogonal to the CMB measurements in the $\Omega_{M}-\Omega_{\Lambda}$ plane, and the curvature measurement at $\mathrm{z} \approx 1$ will test cosmological models by comparison with the CMB determination at $z \approx 1000$. SNAP's science reach will then extend to an exploration of the nature of the dark energy, measuring the present equation of state, $w$, to $5 \%$. Of even more interest is a determination of $w$ as a function of redshift. SNAP's tight control of systematics and high statistics in each redshift bin allows determination of the dynamical variation of $w$.

To complement its supernova cosmology observations, SNAP will conduct a widearea weak lensing survey. These weak lensing observations provide important independent measurements and complementary determinations of the dark matter and dark energy content of the Universe. They will substantially enhance SNAP's ability to constrain the nature of dark energy[11]. SNAP weak lensing observations benefit enormously from the high spatial resolution, the accurate photometric redshifts, and the very high surface density of resolved galaxies available in these deep observations.

Although the SNAP mission is tailored for supernova and weak lensing observations, with the large survey field, depth, spatial resolution, temporal sampling and wavelength coverage into the infrared, the resulting data sets will provide a rich program of auxiliary science. Here we highlight a few selected areas where the large area deep-field observations are expected to significantly impact our understanding of the Universe:

- Galaxies - Within the ultra-deep 15 square degree survey area, SNAP will obtain accurate photometric redshift measurements for at least $5 \times 10^{7}$ galaxies to $z=3.5$. This will provide a unique opportunity to study the evolution of galaxies through more than $90 \%$ of the age of the Universe.

- Galaxy clusters - Galaxy clusters, the most massive bound objects and probably largest structures in the Universe, provide important probes of our understanding of structure formation. The SNAP surveys will provide detailed information on roughly 15,000 galaxy clusters with masses above $5 \times 10^{13} \mathrm{M}_{\odot}$.

- Quasars - NIR photometry extends the redshift range for quasar discovery using colors and dropout surveys. SNAP will be able to detect quasars beyond redshift 10, and to probe the quasar luminosity function to 100 times fainter than the brightest quasars.

- GRBs - Gamma-ray bursts continue to pose a great mystery. Recent observations point to GRBs as the product of core-collapse of super-massive short lived stars. If so, then GRBs may trace the star formation rate and thus GRBs coincident with the epoch of first stars formation are expected. The most distant GRB currently known occurred at redshift of 3.4. SNAP will be able to identify GRB afterglows to $z=10$. 
- Re-ionisation - Most likely the re-ionisation of the Universe did not occur as a single instant in time, but rather as a complex process happening at slightly different epochs in different parts of the Universe. By identifying many quasars and galaxies to $z=10$, SNAP will map the epoch of re-ionization in unprecedented detail.

- Gravitational lensing - The high spatial resolution of SNAP NIR observations will enable the discovery of a large number of new strong lenses. In weak lensing measurements, SNAP spatial resolution and NIR sensitivity will allow the use of a huge number of faint, high-redshift background galaxies. With these galaxies, it will be possible to extend weak lensing studies to lower mass objects, and to study lens objects beyond $z=1$ - measurements which are impossible from the ground.

\section{CONCLUSION}

SNAP presents a unique opportunity to probe the dark energy and advance our understanding of the Universe. It will discover and precisely measure thousands of supernovae of Type Ia and will provide a combination of depth, solid angle and angular resolution heretofore unachieved. From the data collected, it will be possible to precisely measure the equation of state of the Universe, measure the history of its accelerations and decelerations and to study the nature of dark energy, which is causing the current acceleration of the expansion of the Universe. SNAP will be able to measure the equation of state, $w$, of the Universe as well as its variation over time, $w^{\prime}$. This detailed knowledge will allow to distinguish between different models for the nature of the dark energy and lead to decper understanding of the Universe.

\section{ACKNOWLEDGMENTS}

This work was supported by the U.S. Department of Energy.

\section{REFERENCES}

1. Perlmutter, S., et al., Astrophysical Joumal, 517, 565-586(1999).

2. Riess, A., et al., Astronomical Journal, 116, 1009-1038 (1998).

3. Spergel, D. N., et al., astro-ph/0302209 (2003).

4. Bahcall, N. A., et al., Science, 284, 1481 (1999).

5. Ratra, B., and Peebles, P. J. E., Phys. Rev D, 37, 3406-3427 (1988).

6. Caldwell, R. R., Dave, R., and Steinhardt, P., Phys. Rev. Letters, 80, 1582-1585 (1999).

7. Kim, A., et al,, astro-ph/0305286 (2003).

8. Linder, E. V., and Huterer, D., Phys. Rev. D, 67, 081303 (2003).

9. Lampton, M., et al., astro-ph/0209549 (2002).

10. Lampton, M., et al., astro-ph/0210003 (2003).

11. Rhodes, J., et al., astro-ph/0304417 (2003). 